\title{
Análise da correlação entre síndrome de pseudo-exfoliação e aneurisma de aorta abdominal
}

\author{
Analysis of correlation between pseudoex foliation syndrome and aneurysm of the \\ abdominalaorta
}

\author{
Paulo de Tarso Ponte Pierre Filho ${ }^{1}$ \\ Leandro Costa de Araújo ${ }^{2}$ \\ Vital Paulino Costa ${ }^{3}$ \\ Charles Angotti Furtado de Medeiros ${ }^{4}$ \\ George Carchedi Lucas ${ }^{5}$
}

\begin{tabular}{|c|}
\hline RESUMO \\
\hline Objetivo: Examinara correlação sugerida entre síndrome de pseudo-exfolia- \\
ção e aneurisma de aorta abdominal. Métodos: Sessenta e cinco pacientes \\
recentemente operados por aneurisma de aorta abdominal e 51 controles com \\
aterosclerose periférica, sem aneurisma, submeteram-se ao exame oftal- \\
mológico sob dilatação pupilar para investigar a presença de síndrome de \\
pseudo-exfoliação. Resultados: Não houve diferenças estatisticamente \\
significantes entre a média de idade, distribuição por sexo e raça entre os 2 \\
grupos (p > 0,05). Dois dos 65 pacientes com aneurisma de aorta e um dos \\
51 controles apresentaram síndrome de pseudo-exfoliação (p=1,00). Con- \\
clusão: A prevalência de síndrome de pseudo-exfoliação em pacientes \\
operados por aneurisma de aorta abdominal e em pacientes com ateroscle- \\
rose periférica foi similar. Este achado não confirma a associação proposta \\
entre síndrome de pseudo-exfoliação e aneurisma de aorta abdominal.
\end{tabular}

Descritores: Síndrome de exfoliação; Aneurisma da aorta abdominal; Doenças vasculares; Glaucoma; Estudos prospectivos

\section{INTRODUÇÃO}

A síndrome de pseudo-exfoliação (SPEX) é uma fibrilopatia relacionada à idade de causa desconhecida, caracterizada pela deposição de material pseudo-exfoliativo anormal sobre o segmento anterior do olho, além de pele, pulmão, coração, fígado, vasos sanguíneos e meninges ${ }^{(1-3)}$. A síntese ocular e a deposição crônica deste material podem levar a complicações como glaucoma de ângulo aberto e aumento em complicações de cirurgia ocular $^{(1)}$. A associação com oclusão de veia central da retina ${ }^{(4-5)}$ e eventos cardiovasculares $^{(6-7)}$ também tem sido sugerida.

A proporção de pessoas afetadas por essa síndrome variam de 0,5\% em pessoas com idade inferior a 60 anos, a $15 \%$ na população com 60 anos ou mais. Fatores geográficos e étnicos parecem ser importantes, com alta prevalência em escandinavos ${ }^{(8-10)}$ e pacientes de origem mediterrânea ${ }^{(10)}$.

A exata etiologia e patogênese desta síndrome permanecem obscuras. Evidências sugerem que SPEX é um tipo de elastose causada pela síntese excessiva ou degradação reduzida de componentes miofibrilares elásticos, como a fibrilina- $1^{(11)}$.

Uma associação entre aneurismas de aorta abdominal e SPEX foi sugerida ${ }^{(12)}$, que também estaria associada com eventos cardiovasculares $^{(6)}$. Alterações histopatológicas da parede da aorta abdominal em pacientes com SPEX poderiam predispor estes pacientes ao desenvolvimento de aneurismas $^{(12)}$. SPEX, por essa razão, poderia ser um importante marcador 
de risco de doença vascular sistêmica. Neste trabalho, objetivamos verificar a existência da associação clínica entre SPEX e aneurisma de aorta abdominal.

\section{MÉTODOS}

Realizamos um estudo prospectivo caso-controle para diagnóstico de SPEX em 116 pacientes, no período de junho a dezembro de 2002. Dentre esses pacientes, 65 haviam sido submetidos à cirurgia para aneurisma de aorta abdominal e 51 faziam parte de um grupo controle, com aterosclerose periférica. A presença de um índice tornozelo/braço (ITB) $<0,9$ e ausência de quadro clínico sugestivo de aneurisma de aorta abdominal foram os critérios utilizados para inclusão dos indivíduos no grupo controle. Pacientes com idade inferior a 45 anos ou submetidos à cirurgia de catarata bilateral foram excluídos do estudo.

O ITB foi calculado dividindo-se o maior valor da pressão arterial sistólica medida nas artérias tibiais ao nível do tornozelo pelo maior valor da pressão arterial sistólica encontrada nas artérias umerais, pela técnica esfigmomanométrica e com auxílio do Doppler (Parks modelo 1059, Aloha, Oregon).

Em todos os pacientes, foram realizados medida da acuidade visual com tabela de Snellen, biomicroscopia do segmento anterior, medida da pressão intra-ocular com tonômetro de aplanação de Goldmann, gonioscopia com lente de Posner e exame fundoscópico, após dilatação das pupilas com tropicamida $0,5 \%$ e fenilefrina $5 \%$, para avaliação do disco óptico com lente de Volk 78D à lâmpada de fenda. A avaliação do disco óptico foi avaliada subjetivamente pelo examinador, que conferiu aos diâmetros vertical e horizontal da escavação valores que variaram de 0 a 1.

A SPEX foi definida como a presença de material pseudoexfoliativo sobre a cápsula anterior do cristalino ou próximo à pupila, bem como a presença de três dos quatro critérios a seguir: defeitos de transiluminação na porção pupilar da íris, atrofia de liseré, presença da linha de Sampaolesi à gonioscopia e zonas de descontinuidade óptica, pigmentadas, no humor aquoso à midríase medicamentosa ${ }^{(13)}$. Apenas dois médicos realizaram os exames oftalmológicos. Ambos desconheciam a que grupo pertenciam os pacientes.

O estudo foi conduzido de acordo com os termos da Declaração de Helsinque e aprovado pelo Comitê de Ética em Pes- quisa da Faculdade de Ciências Médicas da UNICAMP. Todos os pacientes assinaram um consentimento informado antes da realização do exame.

Para descrever o perfil da amostra segundo as diversas variáveis em estudo, foram feitas tabelas de freqüência das variáveis categóricas e estatísticas descritivas das variáveis contínuas. Realizou-se a equivalência das medidas da acuidade visual entre as escalas de Snellen e Log MAR.

Para comparação das variáveis categóricas entre os grupos, utilizou-se o teste qui-quadrado $\left(\chi^{2}\right)$ ou, quando necessário (valores esperados menores que 5), o teste exato de Fisher. Para comparação das variáveis contínuas entre os grupos foi utilizado o teste de Mann-Whitney. O nível de significância adotado para os testes estatísticos foi de 5\%, ou seja, $p<0,05$. A análise foi feita com o uso do programa SAS (Statistical Analysis System) for Windows, versão 6.12.

\section{RESULTADOS}

Dos 116 pacientes estudados, $95(81,9 \%)$ pertenciam ao sexo masculino e 87 (75\%) eram brancos. A média de idade dos pacientes foi de $67,2 \pm 8,42$ anos. Os dois grupos foram considerados homogêneos em relação ao sexo, idade e raça (Tabela 1).

Em relação aos achados oculares, também não houve diferença significante entre as médias da acuidade visual, pressão intra-ocular e diâmetro da escavação do disco óptico entre os dois grupos. Apenas três $(2,58 \%)$ pacientes tiveram diagnóstico de síndrome de pseudo-exfoliação, sendo dois $(1,72 \%)$ pertencentes ao grupo com aneurisma de aorta abdominal e um $(0,86 \%)$ pertencente ao grupo controle. Todos os três pacientes diagnosticados apresentavam material pseudo-exfoliativo sobre a cápsula anterior do cristalino. Não houve diferença estatisticamente significante entre a frequiência de SPEX entre os grupos $(\mathrm{p}=1,00)$ (Tabela 2).

\section{DISCUSSÃO}

Um estudo caso-controle encontrou associação estatisticamente significante entre SPEX e aneurisma de aorta abdominal ${ }^{(12)}$, detectando a presença de material exfoliativo em 24 (44\%) dos 55 pacientes com aneurisma e em 8 (20\%) dos 41 pacientes com oclusão de artéria carótida $(\mathrm{p}=0,016)$. O exame

\begin{tabular}{|lccc|}
\hline & \multicolumn{2}{c|}{ Tabela 1. Dados demográficos da população estudada } \\
& $\begin{array}{c}\text { Grupo com aneurisma } \\
(\mathbf{n}=\mathbf{6 5})\end{array}$ & $\begin{array}{c}\text { Grupo controle } \\
(\mathbf{n}=\mathbf{5 1})\end{array}$ & $\begin{array}{c}\text { Nível de significância } \\
(\mathbf{p})\end{array}$ \\
Idade (anos) & $67,42 \pm 7,71$ & $66,90 \pm 9,92$ & $\mathrm{p}=0,9025$ \\
Sexo masculino $\mathrm{n}(\%)$ & $56(86,15 \%)$ & $39(76,47 \%)$ & $\mathrm{p}=0,1788$ \\
Sexo feminino $\mathrm{n}(\%)$ & $9(13,85 \%)$ & $12(23,53 \%)$ & $\mathrm{p}=0,5892$ \\
Raça branca $\mathrm{n}(\%)$ & $50(76,92 \%)$ & $37(72,55 \%)$ & \\
Raça negra $\mathrm{n}(\%)$ & $13(20,00 \%)$ & $13(25,49 \%)$ & \\
Raça amarela $\mathrm{n}(\%)$ & $2(3,08 \%)$ & $1(1,96 \%)$ & \\
\hline
\end{tabular}




\begin{tabular}{|c|c|c|c|}
\hline \multicolumn{4}{|c|}{ Tabela 2. Achados oculares } \\
\hline & $\begin{array}{c}\text { Grupo com aneurisma } \\
(n=65)\end{array}$ & $\begin{array}{l}\text { Grupo controle } \\
(n=51)\end{array}$ & $\begin{array}{l}\text { Nível de significância } \\
(p)\end{array}$ \\
\hline AV(Log MAR) (OD) & $0,26 \pm 0,31$ & $0,34 \pm 0,39$ & $p=0,2956$ \\
\hline AV(Log MAR) (OE) & $0,30 \pm 0,40$ & $0,34 \pm 0,37$ & $p=0,3605$ \\
\hline Po (OD) & $14,0 \pm 3,55$ & $14,3 \pm 3,56$ & $p=0,6624$ \\
\hline Po (OE) & $14,1 \pm 3,48$ & $14,9 \pm 3,92$ & $p=0,3429$ \\
\hline DV (OD) & $0,35 \pm 0,17$ & $0,36 \pm 0,19$ & $p=0,5411$ \\
\hline DV (OE) & $0,34 \pm 0,17$ & $0,37 \pm 0,20$ & $p=0,5799$ \\
\hline $\mathrm{DH}(\mathrm{OD})$ & $0,33 \pm 0,15$ & $0,38 \pm 0,19$ & $p=0,5913$ \\
\hline $\mathrm{DH}(\mathrm{OE})$ & $0,34 \pm 0,16$ & $0,36 \pm 0,17$ & $p=0,5540$ \\
\hline SPEX n (\%) & $2(3,08 \%)$ & $1(1,96 \%)$ & $p=1,0000$ \\
\hline
\end{tabular}

histopatológico de cinco amostras de parede aórtica, obtidas de doadores de órgãos com SPEX apresentavam material exfoliativo no tecido conectivo subendotelial e da camada adventícia, fibrose e elastose da túnica íntima. Essas alterações não foram vistas em amostra semelhante na população normal de idade similar.

No entanto, sete dos pacientes com aneurisma que foram incluídos como tendo SPEX tinham o que os autores chamaram "estágio inicial", caracterizado por pobre dilatação pupilar, atrofia de íris e dispersão de grânulos de melanina na câmara anterior. Nenhuma dessas características, contudo, são específicas da SPEX e poderiam ser encontradas na população idosa, independente da presença de doenças oculares ${ }^{(14)}$.

Mais recentemente, depósitos de material pseudo-exfoliativo foram encontrados em apenas $15(19 \%)$ entre 77 pacientes finlandeses operados por aneurismas de aorta abdominal ${ }^{(15)}$. A frequiência de SPEX encontrada foi semelhante à encontrada na população local (controle histórico).

Em nosso estudo, não encontramos associação entre SPEX e aneurisma de aorta abdominal $(\mathrm{p}=1,00)$. Um estudo que avaliou os tipos de glaucoma de uma população seguida no Hospital das Clínicas da UNICAMP encontrou uma prevalência de $1,3 \%$ de glaucoma pseudo-exfoliativo ${ }^{(16)}$. Tal prevalência é inferior à encontrada em outros relatos ${ }^{(6,12,17)}$, provavelmente devido à composição étnica brasileira. A baixa prevalência de SPEX no nosso meio justificaria a necessidade de uma amostra maior de pacientes para que pudéssemos melhor analisar a diferença entre os grupos.

Baseado em nossos achados e em consonância com outro estudo $^{(15)}$, acreditamos que a presença de SPEX em pacientes operados para aneurisma de aorta abdominal no Brasil é semelhante à população geral de mesma idade.

Outra abordagem que nos auxiliaria a investigar a associação entre SPEX e aneurisma de aorta abdominal envolveria o seguimento de dois grupos de pacientes, sendo um deles com SPEX e outro sem, pareados por sexo, idade e status cardiovascular, com ultra-sonografias abdominais seqüenciais para verificar o desenvolvimento de aneurismas ao longo do tempo em ambos os grupos ${ }^{(14)}$.

\section{CONCLUSÃO}

Este estudo não confirmou a associação proposta entre síndrome de pseudo-exfoliação e aneurisma de aorta abdominal. Os estudos nessa área estão apenas começando a serem explorados. Acreditamos que outros estudos, longitudinais ou caso-controle, devam ser realizados para melhor avaliar esta possível associação.

\section{ABSTRACT}

Purpose: To investigate the suggested association between pseudoexfoliation syndrome and aneurysm of the abdominal aorta. Methods: 65 patients recently operated for abdominal aortic aneurysm and 51 controls with peripheral atherosclerosis, without aneurysm, underwent an ophthalmologic examination under pupillary dilatation to detect the presence of pseudoexfoliation syndrome. Results: There were no significant differences regarding mean age and distribution by gender and race between the groups ( $p>0.05$ ). Two of 65 patients with aortic aneurysm and one of 51 controls presented pseudoexfoliation syndrome $(p=1.00)$. Conclusion: The prevalence of pseudoexfoliation syndrome in patients operated for abdominal aortic aneurysm and in patients with peripheral atherosclerosis were similar. This finding does not support the proposed association between pseudoexfoliation syndrome and abdominal aortic aneurysm.

Keywords: Exfoliation syndrome; Aortic aneurism, abdominal; Vascular diseases; Glaucoma; Prospective studies

\section{REFERÊNCIAS}

1. Schlötzer-Scherehardt U, Koca MR, Naumann GO, Volkholz H. Pseudoexfoliation syndrome: ocular manifestation of a systemic disorder? Arch Ophthalmol 1992;110:1752-6.

2. Streeten BW, Dark AJ, Wallace RN, Li ZY, Hoepner JA. Pseudoexfoliative fibrillopathy in the skin of patients with ocular pseudoexfoliation. Am J Ophthalmol 1990;110:490-9. 
3. Streeten BW, Li ZY, Wallace RN, Eagle RC, Keshgegian AA. Pseudoexfoliative fibrillopathy in visceral organs of a patient with pseudoexfoliation syndrome. Arch Ophthalmol 1992;110:1757-62.

4. Cursiefen C, Händel A, Schönherr U, Naumman GO. Das pseudoexfoliation ssyndrom bei Patienten mit retinaler Venenast- und Zentralvenenthrombose. Klin Monatsbl Augenheilkd 1997;211:17-21.

5. Cursifen C, Hammer T, Kückle M, Naumann GOH, Schlötzer-Schrehardt U. Pseudoexfoliation syndrome in eyes with ischemic central retinal vein occlusion. A histopathologic and electron microscopic study. Acta Ophthalmol Scand 2001;79:476-8.

6. Mitchell P, Wang JJ, Smith W. Association of pseudoexfoliation syndrome with increased vascular risk. Am J Ophthalmol 1997;124:685-7.

7. Kling F, Colin J, Joris A. Pseudoexfoliation syndrome (PEX): indicator of increased cardiac and/or vascular risks? [poster]. In: American Academy Ophthalmology; Orlando, Florida, October 1999.

8. Ritch R. Exfoliation syndrome: the most common identifiable cause of openangle glaucoma. J Glaucoma 1994;3:176-8.

9. Ritch R, Schlötzer-Schrehardt U. Exfoliation syndrome. Surv Ophthalmol 2001;45:265-315.

10. Shields MB. Glaucomas associated with disorder of the lens. In: Shields MB.
Textbook of glaucoma. $5^{\text {th }}$ ed. Baltimore: Williams and Wilkins; 1998. p.252-68.

11. Schlötzer-Schrehardt U, Von der Mark K, Sakai Ly, Naumann GO. Increased extracellular deposition of fibrillin-1 containing fibrils in pseudoexfoliation syndrome. Invest Ophthalmol Vis Sci 1997;38:970-84.

12. Schumacher S, Schlötzer-Scherehardt U, Martus P, Lang W, Naumann GOH Pseudoexfoliation syndrome and aneurysms of the abdominal aorta. Lancet 2001;357:359-60.

13. Yüksel N, Karabas L, Arslan A, Demirci A, Caglar Y. Ocular hemodynamics in pseudoexfoliation syndrome and pseudoexfoliation glaucoma. Ophthalmology 2001;108:1043-9.

14. Ringvold A. Pseudoexfoliation and aortic aneurysms. Lancet 2001;357:2139-40.

15. Hietanen J, Soisalon-Soinimen S, Kivelan T, Tarkkanen A. Evaluation the clinical association between exfoliation syndrome and abdominal aortic aneurysm. Acta Ophthalmol Scand 2002;80:617-19.

16. Urbano AP, Freitas TG, Arcieri ES, Urbano AP, Costa VP. Avaliação dos tipos de glaucoma no Serviço de Oftalmologia da UNICAMP. Arq Bras Oftalmol 2003;66:61-5.

17. Ringvold A, Blika S, Elsas T, Guldahl J, Brevik T, Hesstvedt P, et al. The prevalence of pseudoexfoliation in three separate municipalities of Middle-Norway. Acta Ophthalmol 1987; Suppl 182:17-20.

\title{
XXVIII SIMASP - SIMPÓSIO MOACYR E. ÁLVARO
}

\author{
17 a 19 de Fevereiro de 2005 \\ Frei Caneca Shopping \& Convention Center \\ SÃO PAULO - SP
}

"O lho depois dos 40 anos - Do Básico ao A vançado"

Informações: Centro de Estudos de O ftalmologia "Moacyr E. Álvaro" - CEO

Fone: (11) 5085-2026 